\title{
Encuesta de opinión: En pre-grado, 2003-2004 de Patología Oral y Estomatología. La Plata, Argentina
}

\author{
Bruno $\mathrm{M}^{*}$, Dorati P, Casariego $\mathrm{ZJ}^{* *}$
}

\section{RESUMEN}

Se trata de un trabajo de investigación sobre el proceso "enseñanza/aprendizaje" realizado a través de Encuestas de opinión por los alumnos del cuarto y quinto curso de Pregrado, de Patología y Clínica Estomatológica. El objetivo fue el evaluar los resultados del "proceso enseñanza /aprendizaje", y su aprovechamiento por los alumnos.

Material y métodos: Las comisiones de alumnos que entraron en la encuesta fueron tomadas al azar en los cursos lectivos de los años 2003 y 2004. Las preguntas del cuestionario fueron diez, siendo las mismas en cada año. Las respuestas fueron anónimas y se clasificaron por las categorías SI, SIEMPRE, EN GRAN MEDIDA (favorables) y NO, NADA, A VECES, NUNCA Y NO SE RESPONDER (como desfavorables). En este último curso lectivo se agregó una variante pedagógica (multimedia sobre imágenes de patología y estomatología). Se realizó análisis estadístico. Se presentó la información en forma de tablas y gráficos. Se aplicó la prueba del $\chi^{2}$ para comparar cada una de las preguntas realizadas en ambos grupos y en ambos años y se calcularon los odds ratios y sus intervalos de confianza, para indicar el mayor posibilidad favorable para uno u otro grupo, según año de la encuesta.

Resultados: Se observaron diferencias significativas ( $p<0,05$ en 5 de 10 preguntas y altamente significativas $(p<0,01)$ en las 5 restantes, éstas a favor de los casos del año 2003. Los odds ratios también demostraron mayor posibilidad de respuestas favorables para la encuesta del año 2003 que para la del 2004.

Conclusiones: De acuerdo a los resultados obtenidos por el análisis estadístico, podría llegar a inferirse que: 1. Al test aplicar el $\chi^{2}$, los valores hallados demuestran diferencias significativas a favor de las respuestas favorables del año 2003. 2. En cuanto al análisis que surge de la utilización del odds ratio, éste mostró que el proceso enseñanza/aprendizaje tuvo tres veces mejores posibilidades en el año 2003 que en el 2004.

Palabras clave: Encuesta de opinión. Proceso enseñanza/aprendizaje. Autoevaluación pedagógica.

\section{SUMMARY}

This work consist about an investigation of the "teaching and learning process" made trough two surveys of opinion, performed by Pre-grade students of $4^{\text {th }}$ and $5^{\text {th }}$ levels of Oral Pathology and Clinic Stomatology. The objetive was to evaluate Instruments used on "teaching and learning process" and its avail.

Material and methods: The groups of students that took place in answering the questionary were aleatory selected. There were performed same surveys in each year: 2003 and 2004. Answers were anonymous and they were classificated through some categories as: YES, ALWAYS, IN LARGE QUANTITY (favorable), and SOME TIMES, NEVER and I DON"T KNOW TO ANSWER. (as no favorable). Over the last year, a pedagogic variant (multimedia of pathological and stomatologic diseases) was added.

Statistic analys was made. Information was establish on tables and graphics. $\chi^{2}$ test was applied in order to compare each question and i odds ratios was calculated with its confidence intervals well. Its indicated the favorable chance for one or another group of questions, depending the year of each survey. 
Results: Its were observed significant statistic differences $(\mathrm{p}<0.05)$ in 5 of 10 questions and a greatly significant difference $(p<0.01)$ in the other 5 , in favor to year 2003. Odds ratio demostrated more possibility of favorable answers belong 2003 survey as well.

Conclusions: According to results of the two groups of questions and the statistic analysis, it could be inferred that: $1 . \chi^{2}$ test, demostrats significantly statistic differences between favorable and no favorable answers between 2003. and 2004 surveys. 2. About usefulness of odds ratio study, it shaws that "teaching and learning process" in this investigation, has had 3 times better posibilities of evolution in 2003 that in 2004.

Key words: Survey of opinion. Teaching and learning process". Pedagogical autoevaluation.

Aceptado para publicación: Diciembre 2004.

* Docentes ordinarios.

** Profesora Académica.

Bruno M, Dorati P, Casariego ZJ. Encuesta de opinión: En pre-grado, 2003-2004 de Patología Oral y Estomatología. La Plata, Argentina. Av. Odontoestomatol 2006; 22 (3): 187-194.

\section{INTRODUCCIÓN}

A través del asesoramiento de la Cátedra de Bioestadística de la Facultad de Ciencias Veterinarias de la Universidad Nacional de la Plata, se realizó el análisis estadístico de las encuestas realizadas en la asignatura Patología Oral y Clínica y Estomatológica de la facultad de Odontología durante el año 2003 y 2004. La misma fue presentada para su resolución a alumnos de Pre-grado de los cursos cuarto y quinto'.

En general, realizar la evaluación de los aprendizajes sobre el proceso de enseñanza a través de los trabajos prácticos que se realizan durante el año lectivo, en la carrera, y en nuestro caso en especial, es una tarea continua. Sin embargo ésta no es una tarea planeada. A pesar de las pequeñas evaluaciones orales y escritas, la participación de los alumnos en los grupos de trabajos y la visita y observación en terreno de las diferentes patologías, existe la preocupación en el cuerpo docente sobre la eficacia de los métodos utilizados. Más aún cuando se encuentran frente a una evaluación escrita de resultados no muy exitosos. Y así surgen preguntas tales como: si los temas y las actividades propuestas fueron coherentes para el logro de los objetivos, si la metodología utilizada para el desarrollo del programa facilitó la participación del alumno o si los grupos obtuvieron resultados positivos y otra tantas más que hacen al proceso. De modo que utilizando guías para la observación sobre todo del desempeño del docente y de aquel que dirige la enseñanza en general, se puede recurrir a las llamadas "guía de observación del desempeño del docente en clase" o bien a las "encuestas de opinión" utilizadas para evaluar el desempeño del docente recogiendo opiniones de los alumnos sobre el mismo.

Teniendo en cuenta algunos principios orientadores de los alcances de la evaluación educacional en la actualidad es que elegimos la segunda opción mencionada partiendo de los siguientes objetivos, evaluar:

1. El proceso enseñanza /aprendizaje en alumnos de Patología oral y Estomatología de los cursos $4 .^{\circ}$ y $5 .^{\circ}$ durante los años 2003 y 2004.

2. Aprovechamiento de los trabajos prácticos.

3. Utilidad de los apuntes realizados por la Asignatura.

4. Valorar la comprensión tabulando los resultados y extrayendo conclusiones.

\section{MATERIAL Y METODOS}

Debido que en el año 2003 se realizó una encuesta de opinión en alumnos de la Asignatura mencionada, se repitió el cuestionario, con las mismas pre- 
guntas en el actual ciclo lectivo, año 2004, también con carácter anónimo.

Debido a que se agregó el taller de multimedia para la observación de preparados histológicos y casos clínicos, se esperaron resultados más favorables.

Nos ha sido de gran utilidad el análisis estadístico que se aplicó a ambas encuestas con el asesoramiento de la Cátedra de Bioestadística de la Facultad de Ciencias Veterinarias de la Plata.

Se presentó la información en forma de tablas y gráficos, reagrupando respuestas en una clasificación dicotómica (Favorable / Desfavorable) según la tabla 1.

Se adoptó una nueva clasificación de las respuestas modificando la que figuraba en el año 2003 para facilitar el trabajo estadístico (a).

Se aplicó la prueba de $\chi^{2}$ comparando para cada una de las preguntas analizadas respuestas favorables o desfavorables según 2003 y 2004.

Por medio de esta prueba se compararon los datos cualitativos dicotómicos con dos posibles respuestas SI/NO y Favorable y Desfavorable. Medimos así en cuál grupo (2003-2004) hubo mejoría en el proceso enseñanza-aprendizaje, o sea, mayor porcentaje de favorables. Y si las diferencias eran significativas.

a)

\begin{tabular}{|l|l|}
\hline \multicolumn{2}{|c|}{ TABLA 1 } \\
\hline Nueva clasificación & Clasificación original \\
\hline \multirow{2}{*}{ Favorable } & Si \\
& Siempre \\
& En gran medida \\
\hline Desfavorable & No \\
& Nada \\
& A veces \\
& Nunca \\
& No sé responder \\
\hline
\end{tabular}

Se calcularon los odds ratios y sus Intervalos de confianza con un nivel de confianza de $95 \%$, considerando (a fin de construir las tablas) el método clásico de cursada del 2003 como "factor de exposición". El odds ratio con un valor basal de 1 , nos estaba indicando cuánto más favorable era una respuesta que otra. O sea, cuantas más chances de mejoría o favorables eran las opciones del 2003 frente al 2004 o viceversa.

Se realizaron tablas con los valores obtenidos estadísticamente sobre cada uno de los 10 ítems del cuestionario y de los años 2003 y 2004 respectivamente (ver tablas 1-10).

Se tabularon las respuestas de cada pregunta, siempre bajo los códigos de favorable y desfavorable por año, los parciales, el total, el porcentaje, $\chi^{2}$ y OR (ver tablas 1-10) y, se tabuló el porcentaje de respuestas según año favorable y desfavorable como síntesis de la observación de los resultados.

\section{RESULTADOS}

1. No se observaron diferencias significativas $(p>$ $0,05)$ en la pregunta 2.

2. No hubo diferencias significativas entre las respuestas favorables en 2003 y 2004.

3. Se observaron diferencias significativas $(p<0,05)$ en las preguntas 5, 6, 7 y 8 .

4. Se observaron diferencias altamente significativas $(p<0,01)$ en las preguntas $1,3,4,9$ y 10 .

5. Estas diferencias se dieron en todos los casos, a favor del cuestionaron del año 2003.

6. Los odds ratios también demostraran mayor posibilidad de respuestas favorables para el 2003 que para el 2004.

7. El año 2003 presentó un porcentaje mayor de respuestas favorables en todos los ítems que en el año 2004.

\section{DISCUSIÓN}

La acción educativa es un proceso formal y sistemático. Se desarrolla en instituciones quienes deben definir y planificar en qué sentido y de cuál manera van a formar, en nuestro caso, profesionales capa- 
ces. En el ámbito de la Patología Oral y de la Estomatología deberán estar formados para prevenir, diagnosticar, formular esquemas de tratamiento y de rehabilitación para las patologías más frecuentes de nuestro medio. La institución y cada una de las Asignaturas y Cátedras, así como cada uno de los docentes deben decidir el conjunto de actividades formales de aprendizaje que se pondrá a disposición de los alumnos con el fin de modelar su personalidad de acuerdo a los objetivos seleccionados previamente (1).

De acuerdo con J. J. Guilbert (2) "a fin de ser pertinente un programa de enseñanza, en lugar de ser el resultado de una agrupación no selectiva de conocimientos acumulados en el transcurso de los siglos, debería ser estructurado en función del fin a alcanzar" pensamos en el valor de la Evaluación como arma didáctica del proceso Enseñanza /aprendizaje para ir ajustando el mismo de acuerdo a los resultados obtenidos. Sobre esta base y como recurso para que el docente realice una autoevaluación y reflexione sobre su práctica, es que hemos adoptado la forma de "encuesta de opinión" a los alumnos sobre la siguiente base: a) preparación de la encuesta, b) aplicación a no menos de 10 alumnos, tabulamos los resultados y hemos extraído las conclusiones para continuar o modificar las estrategias de enseñanza utilizadas en los dos períodos de tiempo encuestados. Nos hemos guiado, para tal propósito, por el programa de Formación de Formadores en Ciencias de la Salud y sobre los conceptos Básicos de Evaluación Educacional $(3,4,5)$.

Consideramos sin embargo que, los problemas de orden externo como ser grupos numerosos de alumnos, ambientes reducido de trabajo, falencias en la educación intermedia preuniversitaria, hacen a resultados desfavorables, y que éstos se relacionan con los contenidos de las políticas gubernamentales, el cambio de costumbres sociales, el déficit de programas televisivos formativos, etc. Que se dan en muchos países, en nuestro tiempo $(6,7)$.

\section{CONCLUSIONES}

De acuerdo al análisis de los resultados obtenidos sobre las preguntas contestadas en estas dos en-
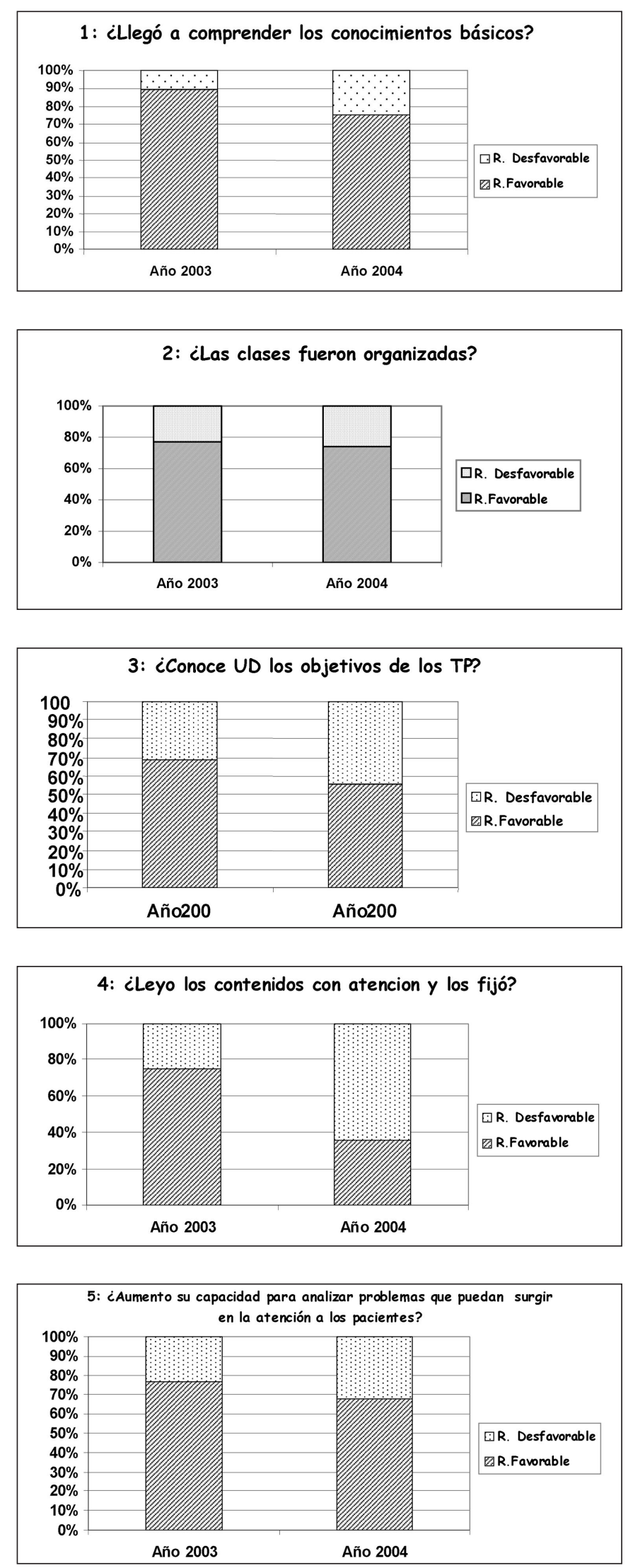

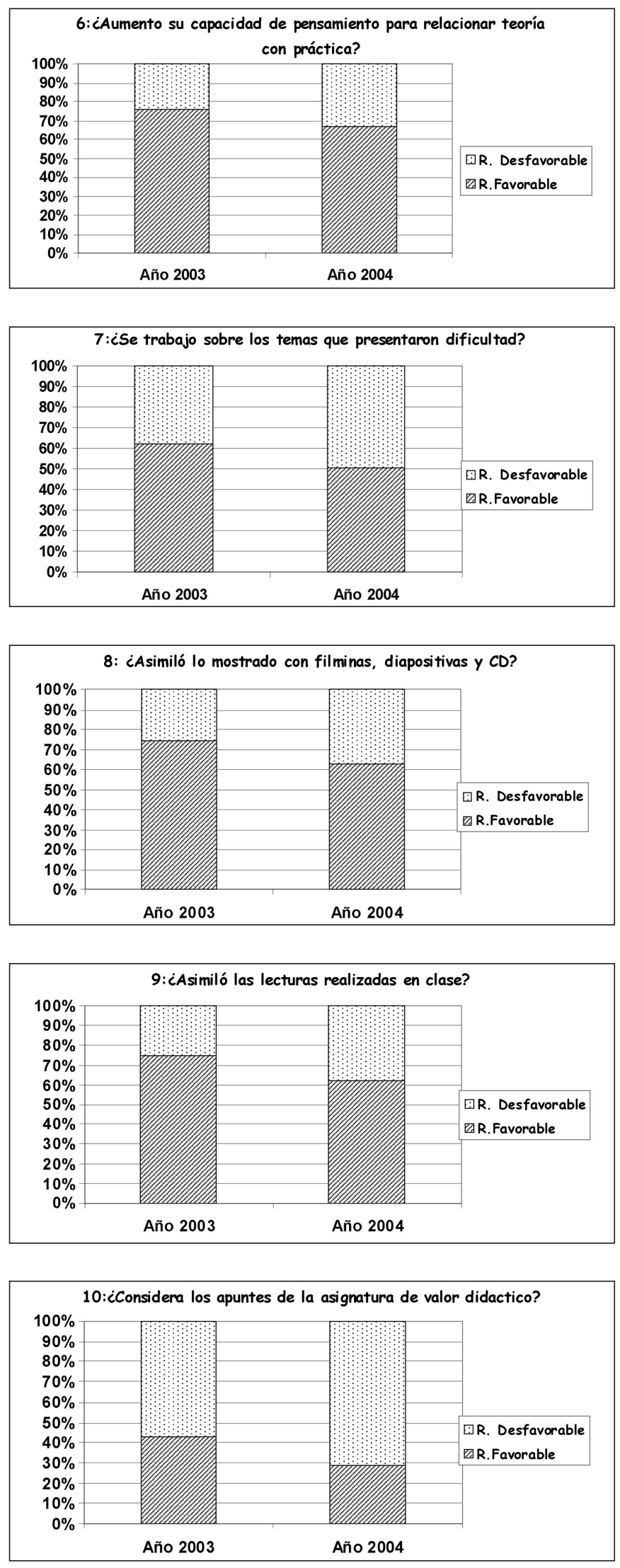

cuestas, podría llegar a inferirse sobre el análisis estadístico:

1. Al aplicar el test del $\chi^{2}$ la comparación marca la diferencia significativa para las respuestas favorables del año 2003 (En este caso podríamos descartar la probabilidad del azar o causalidad ya que ésta podría ser significativa cada 100 veces en 5, lo cual no ha ocurrido).

2. En cuanto al estudio del odds ratio, éste mostró que el proceso enseñanza/aprendizaje tuvo tres veces mejores posibilidades en el año 2003 que en el 2004.

3 . Teniendo en cuenta las dos conclusiones anteriores, podríamos inferir que, tanto el profesor superior como cada docente, debería realizar una autoevaluación del proceso ya que el alumno pareciera estar acostumbrado al método de la "exposición”. El método de observación de imágenes por multimedia se estaría comportando, en este caso, como un "distractor", por lo cual habría que modificar la forma de su aplicación.

El aprovechamiento de los "apuntes" pareciera ser un obstáculo y no un beneficio para los alumnos de los cursos lectivos del corriente año, pues parecen ser de un nivel demasiado elevado para el alumno medio.

\section{BIBLIOGRAFÍA}

1. Bertoni A, Poggi M, Teobaldo M. Evaluación. Nuevos significados para una práctica completa. Kapelutz, Buenos Aires, 1996.

2. Guilbert JJ. Guía pedagógica para el personal de salud. OPS/OMS, Ginebra 1980.

3. Amanda Galli; Programa de Formación de formadores en Ciencias de la Salud- Prensa m;édica Argentina, Buenos Aires, 1999.

4. Santos guerra MA.:Evaluación Educativa. Ed Magisterio del Río de La Plata.Buenos Aires 1996.

5. Stillman P, etal.: An Assesment of clinical skills of fourth year students at Four New England medical School Academic medicine. 1990, 65: 320-326. 
6. Amanda Galli: El proceso de Enseñanza y Aprendizaje: Módulo 5. Paltex. OPS?OMS 1992.

7. Shön DA. La formación de profesionales reflexivos. Hacia un nuevo diseño de la enseñanza y el aprendizaje en las profesiones. Paidós, Barcelona, 1992.

\section{CORRESPONDENCIA}

Prof. Dra. Z. J. Casariego

Bte. Mitre 1371-4M-1036

Buenos Aires-Argentina

FAX+ 0054-11-43720444

E-Mail: zulemacasariego@hotmail.com

\section{TRANSCRIPCION DE LA ENCUESTA DE OPINIÓN QUE SE PRESENTÓ A LOS ALUMNOS PARA LA ENCUESTA}

USTED PUEDE RESPONDER CON: Si-no-nada-siempre-a veces nunca-EGM (en gran medida)-NSR (no sé responder).

Esta encuesta tiene valor didáctico para realizar una valoración del proceso enseñanza-aprendizaje. Colabore contestando con seriedad. Colabore en forma anónima.

1. ¿LLEGÓ USTED A COMPRENDER LOS CONOCIMIENTOS BASICOS?

2. ¿LAS CLASES FUERON ORGANIZADAS?

3. ¿CONOCE USTED LOS OBJETIVOS DE LOS T.P.?

4. ¿LEYÓ LOS CONTENIDOS CON ATENCIÓN Y LOS FIJO?

5. ¿AUMENTÓ SU CAPACIDAD PARA ANALIZAR PROBLEMAS QUE PUEDAN SURGIR EN LA ATENCION A LOS PACIENTES?

6. ¿AUMENTÓ SU CAPACIDAD DE PENSAMIENTO PARA RELACIONAR LA TEORIA CON LA PRACTICA?

7. ¿SE TRABAJO SOBRE LOS TEMAS QUE PRESENTARON DIFICULTAD?

8. ¿ASIMILÓ LO MOSTRADO CON FILMINAS, DIAPOSITIVOS Y CDs?

9. ¿ASIMILÓ LAS LECTURAS REALIZADAS EN CLASE?

10. ¿CONSIDERA LOS APUINTES DE LA ASIGNATURA DE VALOR DIDÁCTICO?

\begin{tabular}{|c|cc|cc|}
\hline \multicolumn{4}{|c|}{ PORCENTAJE DE RESPUESTAS SEGÚN AÑO } \\
\hline \multirow{2}{*}{$\begin{array}{c}\text { Núm. de } \\
\text { ítems }\end{array}$} & \multicolumn{2}{|c|}{ Año 2003 } & \multicolumn{2}{c|}{ Año 2004 } \\
\hline $\mathbf{1}$ & \% Favorables & \% Desfavorables & \% Favorables & \% Desfavorables \\
\hline $\mathbf{2}$ & 90 & 10 & 75 & 25 \\
\hline $\mathbf{3}$ & 77 & 23 & 74 & 26 \\
\hline $\mathbf{4}$ & 69 & 31 & 55 & $\mathbf{4 5}$ \\
\hline $\mathbf{5}$ & 75 & 25 & 36 & 32 \\
\hline $\mathbf{7}$ & 77 & 23 & 68 & 33 \\
\hline $\mathbf{8}$ & 75 & 25 & 67 & 49 \\
\hline $\mathbf{9}$ & 63 & 37 & 51 & 37 \\
\hline $\mathbf{1 0}$ & 74 & 26 & 63 & 38 \\
\hline
\end{tabular}




\section{PATOLOGía y CLÍNICA ESTOMATOLÓGICA}

ENCUESTA 2003 (208 encuestados)-2004 (231 encuestados).

RESPIESTAS: SI, siempre, EGM se tomaron como favorables.

RESPUESTAS: No, nada, nunca, A veces, NSR se tomaron como desfavorables.

\begin{tabular}{|c|c|c|c|c|c|c|}
\hline \multirow[t]{2}{*}{ Pregunta 1} & \multicolumn{6}{|c|}{ ¿Llegó usted a comprender los conocimientos básicos? } \\
\hline & R. Favorable & R. Desfavorable & Total & $\%$ favorables & $\begin{array}{l}\chi^{2}= \\
\text { LS 95\%: }\end{array}$ & $\begin{array}{c}15,9 \\
5,01\end{array}$ \\
\hline Año 2003 & 187 & 21 & 208 & 90 & $\mathrm{OR}=$ & 2,92 \\
\hline Año 2004 & 174 & 57 & 231 & 75 & LI 95\%: & 1,70 \\
\hline \multirow[t]{2}{*}{ PREGUNTA 2} & \multicolumn{6}{|c|}{ ¿Las clases fueron organizadas? } \\
\hline & R. Favorable & R. Desfavorable & Total & $\%$ favorables & $\begin{array}{l}\chi^{2}= \\
\text { LS 95\%: }\end{array}$ & $\begin{array}{l}0,5 \\
1,81\end{array}$ \\
\hline Año 2003 & 160 & 48 & 208 & 77 & $\mathrm{OR}=$ & 1,17 \\
\hline Año 2004 & 171 & 60 & 231 & 74 & LI 95\%: & 0,76 \\
\hline \multirow[t]{2}{*}{ PREGUNTA 3} & \multicolumn{6}{|c|}{ ¿Conoce UD los objetivos de los TP? } \\
\hline & R. Favorable & R. Desfavorable & Total & $\%$ favorables & $\begin{array}{l}\chi^{2}= \\
\text { LS 95\%: }\end{array}$ & $\begin{array}{l}8,2 \\
2,62\end{array}$ \\
\hline Año 2003 & 143 & 65 & 208 & 69 & $\mathrm{OR}=$ & 1,77 \\
\hline Año 2004 & 128 & 103 & 231 & 55 & LI 95\%: & 1,20 \\
\hline \multirow[t]{2}{*}{ Pregunta 4} & \multicolumn{6}{|c|}{ ¿Leyó los contenidos con atencion y los fijó? } \\
\hline & R. Favorable & R. Desfavorable & Total & $\%$ favorables & $\begin{array}{l}\chi^{2}= \\
\text { LS 95\%: }\end{array}$ & $\begin{array}{c}67,4 \\
8,09\end{array}$ \\
\hline Año 2003 & 156 & 52 & 208 & 75 & $\mathrm{OR}=$ & 5,35 \\
\hline Año 2004 & 83 & 148 & 231 & 36 & LI 95\%: & 3,54 \\
\hline \multirow[t]{2}{*}{ Pregunta 5} & $\begin{array}{l}\text { ¿Aumen } \\
\text { surgir }\end{array}$ & $\begin{array}{l}\text { su capacidad para } \\
\text { la atención a los } \mathrm{p}\end{array}$ & $\begin{array}{l}\text { alizar p } \\
\text { entes? }\end{array}$ & emas que puedan & & \\
\hline & R. Favorable & R. Desfavorable & Total & $\%$ favorables & $\begin{array}{l}\chi^{2}= \\
\text { LS 95\%: }\end{array}$ & $\begin{array}{l}4,4 \\
2,40\end{array}$ \\
\hline Año 2003 & 160 & 48 & 208 & 77 & $\mathrm{OR}=$ & 1,57 \\
\hline Año 2004 & 157 & 74 & 231 & 68 & LI 95\%: & 1,03 \\
\hline
\end{tabular}




\begin{tabular}{|c|c|c|c|c|c|c|}
\hline \multirow[t]{2}{*}{ Pregunta 6} & \multicolumn{6}{|c|}{ ¿Aumentó su capacidad de pensamiento para relacionar teoría con práctica? } \\
\hline & R. Favorable & R. Desfavorable & Total & $\%$ favorables & $\begin{array}{l}\chi^{2}= \\
\text { LS 95\%: }\end{array}$ & $\begin{array}{l}4,1 \\
2,34\end{array}$ \\
\hline Año 2003 & 157 & 51 & 208 & 75 & $\mathrm{OR}=$ & 1,54 \\
\hline Año 2004 & 154 & 77 & 231 & 67 & LI 95\%: & 1,01 \\
\hline \multirow[t]{2}{*}{ Pregunta 7} & \multicolumn{6}{|c|}{ ¿Se trabajó sobre los temas que presentaron dificultad? } \\
\hline & R. Favorable & R. Desfavorable & Total & $\%$ favorables & $\begin{array}{l}\chi^{2}= \\
\text { LS 95\%: }\end{array}$ & $\begin{array}{l}6,2 \\
2,38\end{array}$ \\
\hline Año 2003 & 130 & 78 & 208 & 63 & $\mathrm{OR}=$ & 1,62 \\
\hline Año 2004 & 117 & 114 & 231 & 51 & LI 95\%: & 1,11 \\
\hline \multirow[t]{2}{*}{ Pregunta 8} & \multicolumn{6}{|c|}{ ¿Asimiló lo mostrado con filminas, diapositivas y CD? } \\
\hline & R. Favorable & R. Desfavorable & Total & $\%$ favorables & $\begin{array}{l}\chi^{2}= \\
\text { LS 95\%: }\end{array}$ & $\begin{array}{l}6,4 \\
2,55\end{array}$ \\
\hline Año 2003 & 154 & 54 & 208 & 74 & $\mathrm{OR}=$ & 1,69 \\
\hline Año 2004 & 145 & 86 & 231 & 63 & LI 95\%: & 1,12 \\
\hline \multirow[t]{2}{*}{ Pregunta 9} & \multicolumn{6}{|c|}{ ¿Asimiló las lecturas realizadas en clase? } \\
\hline & R. Favorable & R. Desfavorable & Total & $\%$ favorables & $\begin{array}{l}\chi^{2}= \\
\text { LS 95\%: }\end{array}$ & $\begin{array}{l}8,1 \\
2,74\end{array}$ \\
\hline Año 2003 & 156 & 52 & 208 & 75 & $\mathrm{OR}=$ & 1,81 \\
\hline Año 2004 & 144 & 87 & 231 & 62 & LI 95\%: & 1,20 \\
\hline \multirow[t]{2}{*}{ PREgunta 10} & ¿Consic & los apuntes de la & gnatur & valor didáctico & & \\
\hline & R. Favorable & R. Desfavorable & Total & $\%$ favorables & $\begin{array}{l}\chi^{2}= \\
\text { LS } 95 \% \text { : }\end{array}$ & $\begin{array}{l}9,1 \\
2,72\end{array}$ \\
\hline Año 2003 & 89 & 119 & 208 & 43 & $\mathrm{OR}=$ & 1,83 \\
\hline Año 2004 & 67 & 164 & 231 & 29 & LI 95\%: & 1,23 \\
\hline
\end{tabular}

